\title{
IDENTIFICATION OF SYNCHRONOUS GENERATOR TRANSFER FUNCTIONS FROM STANDSTILL TEST DATA ${ }^{1}$
}

\author{
David-P. Molenaar, O.H. Bosgra \\ Mechanical Engineering \\ Systems and Control Group \\ Delft University of Technology \\ Mekelweg 2 \\ 2628 CD Delft \\ The Netherlands \\ D.P.Molenaar@wbmt.tudelft.nl
}

\author{
M.J. Hoeijmakers \\ Electrical Power Processing Group \\ Faculty of Information Technology and Systems \\ Delft University of Technology \\ Mekelweg 4 \\ 2628 CD Delft \\ The Netherlands \\ M.J.Hoeijmakers@its.tudelft.nl
}

\begin{abstract}
The design of a robust frequency converter controller for high dynamic performance of a synchronous generator requires an accurate dynamic model of the electromagnetic part. In this paper a new procedure for identifying the transfer functions of Park's $d q$-axis model of a synchronous generator has been developed. It will be shown that the parameters of this model can be easily identified from standstill time-domain data. The validity of the theoretical model has been verified by comparing time-domain simulations with measurements taken from the Lagerwey LW-50/750 directdrive synchronous generator. It can be concluded that a consistent model estimate of the electromagnetic part of the LW-50/750 generator has been obtained. Ultimate validation, however, will follow after the implementation of the designed frequency converter controller in this wind turbine.
\end{abstract}

\section{INTRODUCTION}

Recent advances in power electronics applied to wind turbines have drawn the attention to turbines which possess the ability to continuously vary rotational speed with the wind velocity, although world wide they are still outnumbered by constant rotational speed wind turbines. Constant speed operation has not been the choice of wind turbine designers, but rather a necessity brought about by the fixed relationship between the speed of the AC generators and the fixed utility grid frequency. The two main advantages of variable speed operation over constant speed operation are additional energy capture at partial load and potential reduction of fatigue loads.

Fatigue load reduction is the most important and underestimated advantage of variable rotational speed operation. $\mathrm{Ob}$ -

\footnotetext{
${ }^{1}$ Copyright (C) 2002 by Delft University of Technology. Published by the American Institute of Aeronautics and Astronautics, Inc. and the American Society of Mechanical Engineers with permission.
}

viously, it requires high bandwidth control. The bandwidth of the active blade pitch system is, in general, too small to achieve fatigue load reduction. As a result, pitch control has to be used to follow minute-to-minute fluctuations in aerodynamic power, while the electromechanical torque control will focus on fatigue load reduction.

The design of such a robust frequency converter controller for high dynamic performance requires that the (synchronous) generator model parameters are known accurately. In principle, synchronous machine parameters may be determined either from design calculations or from measurements acquired at the factory or on site. For high dynamic performance control, however, the former approach is inadequate. A primary goal of this paper is to address the latter issue.

Many papers have been published on synchronous machine parameter identification ${ }^{1,5,7,13,15,16}$ (and references therein). Most papers address standstill frequency response (SSFR) methods following the protocols of IEEE Standard $115-1995^{4}$. This standard focuses on identifying equivalent circuit parameters rather than on transfer functions. A few papers address methods of identifying the parameters from time-domain data. In both cases, the parameter estimation process generally consists of two parts. First, the time constants are extracted by applying a curve-fitting procedure to measured data. Next, the equivalent circuit parameters are determined by solving a set of non-linear equations through numerical optimization. The weakness of this approach is that the order of the model must be known before the parameters can be determined and that numerical optimization is a process fraught with numerical difficulties ${ }^{3}$.

In this paper a new procedure is developed (using ideas from Touhami et al. ${ }^{15}$ ) for identifying the transfer functions of Park's $d q$-axis model of a synchronous generator from timedomain standstill step-response data. The contribution of 
this paper is that Park's $d q$-axis model equations are rewritten such that a model structure arises that can be easily translated into a simulation scheme. The order of the rational transfer functions is not fixed but is determined by the data. The combination of identifying rational transfer functions and a high signal-to-noise ratio resulting from a standstill test offers the possibility to analytically determine the model parameters. That is, the identification procedure does not require good initial parameter values.

The resulting model is intended to be used for the design of a (robust) frequency converter controller that maximises the energy capture while minimising the fatigue loads of a wind turbine. The newly developed identification procedure will be applied to a $750 \mathrm{~kW}$ direct-drive synchronous generator implemented in the Lagerwey LW-50/750 wind turbine. This paper does not address the problem of saturation because the generator under investigation does not exhibit saturation under normal operating conditions.

The structure of this paper is as follows. Section II treats the topic of synchronous generator modeling. Next, in Section III, a new identification procedure will be presented. In Section IV the developed identification procedure is used to identify the dynamic behaviour of the Lagerwey LW-50/750 generator. Section V presents the conclusions, and recommendations of this paper.

\section{SYNCHRONOUS GENERATOR MODELING}

The aim of this section is to set up a theoretical model of a synchronous generator suited for both time-domain simulation, and model based control design. In essence, there are two aspects of a synchronous generator that need to be modeled, viz. the (electro)mechanical and the electromagnetic part. The mechanical part can be modeled using the techniques outlined in Molenaar ${ }^{9}$. In the present paper we will restrict ourselves to the dynamic modeling of the electromagnetic part.

From a modeling point of view all synchronous generators have similar representations. They differ only with respect to some model parameters. Because the round-rotor synchronous generator is a special case of the salient-pole rotor synchronous generator, we will treat only the latter for an arbitrary number of pole-pairs $p$. Fig. 1 depicts a salient-rotor synchronous generator with only one pole-pair $(p=1)$. The machine has the usual three stator windings, each 120 (electrical) degrees apart. The stator windings are star connected. The rotor has one accessible circuit, the field or excitation winding, and two sets of inaccessible circuits, called damper windings. Damper windings are real or fictitious windings that can be used to represent for example the damping effects of eddy currents in the machine. In Fig. 1, one damper winding is located along the direct-axis, and one along the quadrature-axis (represented by $\psi_{1 d}$ and $\psi_{1 q}$ in Fig. 1). When the DC excitation of the field winding is provided by permanent magnets, the field windings can be replaced by fictitious ones carrying constant field currents.

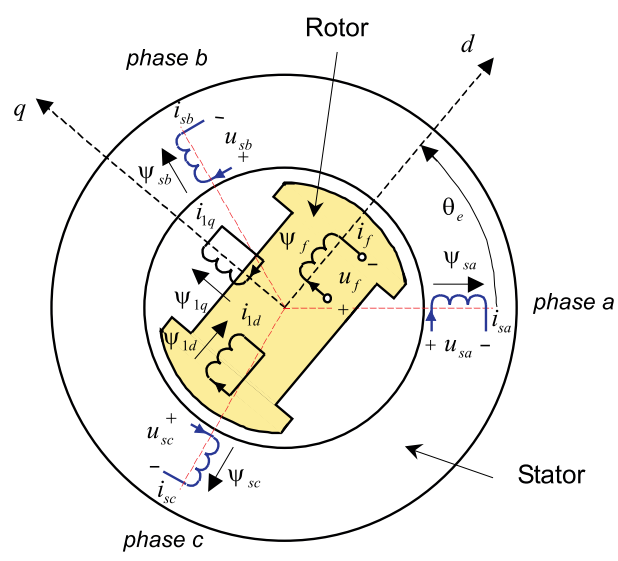

Figure 1: Schematic representation of an elementary three-phase, two-pole synchronous generator.

According to Park ${ }^{11,12}$ the voltage equations of an ideal synchronous generator (i.e. linear magnetic circuit and stator windings are sinusoidally distributed along the stator circumference) in the $d q$ reference frame are given by (using generator sign convention for the stator circuits):

$$
\begin{aligned}
u_{d} & =-R_{s} i_{d}-\omega_{e} \psi_{q}-\frac{d}{d t} \psi_{d} \\
u_{q} & =-R_{s} i_{q}+\omega_{e} \psi_{d}-\frac{d}{d t} \psi_{q} \\
-u_{f} & =-R_{f} i_{f}-\frac{d}{d t} \psi_{f}
\end{aligned}
$$

with $u_{d}$ the direct-axis voltage [V], $R_{s}$ the stator-winding resistance $[\Omega], i_{d}$ the direct-axis current $[\mathrm{A}], \omega_{e}=\frac{d \theta_{e}}{d t}$ the electrical angular frequency $[\mathrm{rad} / \mathrm{s}], \psi_{q}$ the quadrature-axis winding flux [Vs], $t$ time [s], $\psi_{d}$ the direct-axis winding flux [Vs], $u_{q}$ the quadrature-axis voltage [V], $i_{q}$ the quadratureaxis current $[\mathrm{A}], u_{f}$ the field-winding voltage $[\mathrm{V}], R_{f}$ the field-winding resistance $[\Omega], i_{f}$ the field-winding current [A], and $\psi_{f}$ the field-winding flux [Vs].

A few observations can be made. The most important one is that equations (1) are coupled via the fluxes. In addition, they depend on the electrical angular frequency $\omega_{e}$, thereby introducing non-linearities.

The fluxes are given by

$$
\begin{aligned}
& \Psi_{d}(s)=L_{d o}(s) I_{d}(s)+L_{d f o}(s) I_{f}(s) \\
& \Psi_{q}(s)=L_{q}(s) I_{q}(s) \\
& \Psi_{f}(s)=L_{f d o}(s) I_{d}(s)+L_{f o}(s) I_{f}(s)
\end{aligned}
$$

with $s$ is the Laplace operator and $L_{d o}(s), L_{f d o}(s)=$ $L_{d f o}(s), L_{q}(s), L_{f o}(s)$ proper transfer functions (i.e. $\lim _{s \rightarrow \infty} L(s)$ is a finite (zero or non-zero) constant) which depend on the design of the generator. The aforementioned 
transfer functions are in literature often referred to as "operational inductances".

A few comments have to be made. For a finite number of damper windings the aforementioned transfer functions can be expressed as a ratio of polynomials in $s^{6}$. Furthermore, in his original paper R.H. Park used the non-power invariant transformation to transform the stator quantities onto the $d q$ reference frame that is fixed to the rotor. In the above derivation we have used the power-invariant version in order to ensure that in both reference frames the same power expressions are obtained. In addition, he used motor sign convention for the stator circuits.

The dynamic behaviour of an ideal synchronous generator is thus fully described by the sets of equations (1) and (2) expressed in the $d q$ reference frame. For time-domain simulation purposes, it is convenient to rewrite the first set of equations in the following form

$$
\begin{aligned}
& \psi_{d}=-\int\left(u_{d}+R_{s} i_{d}+\omega_{e} \psi_{q}\right) d t \\
& \psi_{q}=-\int\left(u_{q}+R_{s} i_{q}-\omega_{e} \psi_{d}\right) d t \\
& \psi_{f}=\int\left(u_{f}-R_{f} i_{f}\right) d t
\end{aligned}
$$

with the fluxes as state variables. The aforementioned rotor flux equations can be conveniently expressed in matrix form

$$
\left[\begin{array}{l}
\Psi_{d} \\
\Psi_{f}
\end{array}\right]=\left[\begin{array}{cc}
L_{d o}(s) & L_{f d o}(s) \\
L_{f d o}(s) & L_{f o}(s)
\end{array}\right] \cdot\left[\begin{array}{c}
I_{d} \\
I_{f}
\end{array}\right]
$$

It can be easily shown that the inverse transformation is given by

$$
\left[\begin{array}{l}
I_{d} \\
I_{f}
\end{array}\right]=\frac{\left[\begin{array}{cc}
L_{f o}(s) & -L_{f d o}(s) \\
-L_{f d o}(s) & L_{d o}(s)
\end{array}\right]}{L_{d o}(s) \cdot L_{f o}(s)-L_{f d o}^{2}(s)} \cdot\left[\begin{array}{l}
\Psi_{d} \\
\Psi_{f}
\end{array}\right]
$$

In addition, it can be shown that the denominators of $L_{d o}(s)$, $L_{d f o}(s), L_{f d o}(s)$, and $L_{f o}(s)$ are identical. Consequently, the denominators in the above matrix equation are identical. The resulting block diagram is depicted in Fig. 2 for the standstill case (i.e. $\left.\omega_{e}=0\right)$.

Obviously, for simulation as well as control design purposes, accurate information about the transfer functions $L_{d o}(s)$, $L_{f d o}(s), L_{q}(s)$ and $L_{f o}(s)$ as well as the resistances $R_{s}$ and $R_{f}$ is required.

\section{PARAMETER IDENTFICATION}

Synchronous machine identification and parameter determination can be performed either during normal operation (i.e. on-line), or during specially designed identification experiments (i.e. off-line). Each approach has advantages as well

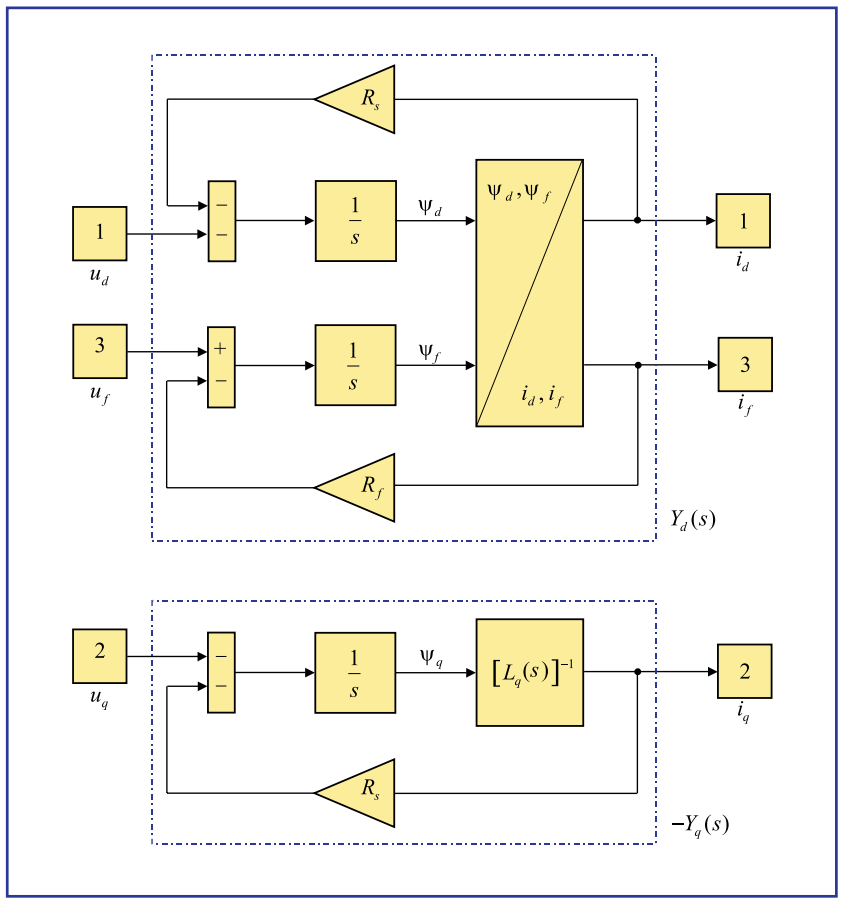

Figure 2: Block diagram of an ideal synchronous machine at standstill.

as disadvantages. For example, in the on-line case the measured input-output data reflect the actual operating conditions. A disadvantage is that it is not possible to manipulate the input signals arbitrarily in order to obtain the best identification results. This implies that the influence of the operating conditions on the (accuracy of the) identified parameters has to be carefully analysed. Off-line tests, on the other hand, require that the machine under test has to be taken out of regular operation, what might be inappropriate or even impossible.

Off-line identification experiments have been used for decades to identify electrical machine parameters. Two concepts are commonly used: running machine, or standstill. Standstill test are very attractive from a practical viewpoint - if it is allowed to take the machine out of operation - because driving the machine is often a serious complication of the measurement set-up 17 . Furthermore, the measured signals will have good signal-to-noise ratios due to the absence of disturbance signals (electromagnetic interference). All standstill tests reported in literature are variations on the same concept, they mainly differ in the kind of excitation signal applied (i.e. step, ramp, sinusoidal, or random excitation).

The standstill test concept is preferred because there is no interaction between the direct- and the quadrature axis. Using this observation, it can be concluded that the parameter identification for both axes may be carried out separately. In practice, zero generator speed can be enforced by mechanically locking the rotor during the experiments. 
In addition, there are a few important practical demands in selecting a technique for the identification and parameter determination of the Lagerwey LW-50/750 generator considering the fact that the generator has already been installed in the nacelle at about 50 meter above the ground level. First of all, the measurements should have low power consumption. Secondly, the test equipment should be compact, and finally, the measurement time should be limited (i.e. $<1$ day).

Before selecting the most appropriate standstill test, we will first highlight the most important aspects of both the quadrature-axis and the direct-axis identification.

\section{A. Quadrature-axis identification}

The dynamic behaviour of the quadrature-axis of an ideal synchronous generator is fully described by the transfer function $Y_{q}(s)$ in Fig. 2. From the block diagram it directly follows that

$$
Y_{q}(s)=-\frac{I_{q}(s)}{U_{q}(s)}=\frac{1}{R_{s}+s \cdot L_{q}(s)}
$$

For the identification of $Y_{q}(s)$ knowledge of the quadratureaxis voltage $u_{q}(t)$ and current $i_{q}(t)$ is thus both necessary and sufficient. It will be shown below that these quantities can be easily derived from three measurable variables, viz. the stator voltages of the $b$ and $c$ phase $\left(u_{b}(t)\right.$, and $u_{c}(t)$ respectively), and the stator current $i_{c}(t)$.

An appropriate rotor position for quadrature-axis identification is the one when the field winding axis is parallel to the a-phase winding (i.e. $\theta_{e}=0$, see Fig. 1). In addition, if in this position the stator $b-c$ terminals are excited while the $a$ terminal remains open (i.e. $i_{a}=0$ ) it follows that $i_{b}=-i_{c}$. Furthermore, it can be concluded from symmetry considerations that $u_{a}=\frac{1}{2}\left(u_{b}+u_{c}\right)$.

Substituting the above results in the equations for the transformed stator voltages and currents

$$
\begin{aligned}
\mathbf{u}_{\text {odq }} & =\mathbf{T}_{\text {odq }} \cdot \mathbf{u}_{s} \\
\mathbf{i}_{\text {odq }} & =\mathbf{T}_{\text {odq }} \cdot \mathbf{i}_{s}
\end{aligned}
$$

where

$$
\begin{aligned}
\mathbf{u}_{\text {odq }} & =\left[\begin{array}{lll}
u_{o} & u_{d} & u_{q}
\end{array}\right]^{T} \\
\mathbf{u}_{\mathbf{s}} & =\left[\begin{array}{lll}
u_{a} & u_{b} & u_{c}
\end{array}\right]^{T} \\
\mathbf{i}_{\text {odq }} & =\left[\begin{array}{lll}
i_{o} & i_{d} & i_{q}
\end{array}\right]^{T}
\end{aligned}
$$

and $\mathbf{T}_{o d q}$ the Park's power-invariant transformation matrix, $\mathbf{T}_{o d q}=\sqrt{\frac{2}{3}}\left[\begin{array}{ccc}\frac{1}{\sqrt{2}} & \frac{1}{\sqrt{2}} & \frac{1}{\sqrt{2}} \\ \cos \left(\theta_{e}\right) & \cos \left(\theta_{e}-\frac{2}{3} \pi\right) & \cos \left(\theta_{e}+\frac{2}{3} \pi\right) \\ \sin \left(\theta_{e}\right) & \sin \left(\theta_{e}-\frac{2}{3} \pi\right) & \sin \left(\theta_{e}+\frac{2}{3} \pi\right)\end{array}\right]$

gives

$$
\begin{array}{ll}
u_{q}=\frac{1}{2} \sqrt{2}\left(u_{c}-u_{b}\right) & u_{d}=0 \\
i_{q}=\sqrt{2} i_{c} & i_{d}=0
\end{array}
$$

Finally, the $q$-axis parameters (i.e. $L_{q}(s)$ and $R_{s}$ ) are deduced algebraically from the transfer function $Y_{q}(s)$.

\section{B. Direct-axis identification}

The dynamic behaviour of the direct-axis of an ideal synchronous generator is fully described by the transfer function matrix $Y_{d}(s)$ between $u_{d}, u_{f}$ and $i_{d}, i_{f}$ in Fig. 2. In this case, an appropriate rotor position is the one when the field winding axis is perpendicular to the a-phase winding (i.e. $\theta_{e}=\frac{1}{2} \pi$, see Fig. 1). After all, it can be easily shown that for $\theta_{e}=\frac{1}{2} \pi$ it follows that

$$
\begin{array}{ll}
u_{d}=-\frac{1}{2} \sqrt{2}\left(u_{c}-u_{b}\right) & u_{q}=0 \\
i_{d}=-\sqrt{2} i_{c} & i_{q}=0
\end{array}
$$

In principle, the elements of Eq. (5), viz. $L_{f o}(s), L_{f d o}(s)$ and $L_{d o}(s)$, can be identified using data acquired from two independent measurements, namely one with excitation of the quadrature-axis voltage while the field winding is left open and one when the field winding is short-circuited ${ }^{17}$. Combining the resulting transfer functions gives the required $2 \times 2$ transfer function matrix. Due to the finiteprecision arithmetic of a computer, however, this will result in an ill-conditioned matrix.

One way to overcome this problem is to identify the MIMO (multiple-input-multiple-output) transfer function between the fluxes $\psi_{d}, \psi_{f}$ and the currents $i_{d}, i_{f}$ assuming that both $R_{s}$ and $R_{f}$ are known. Recall that the stator winding resistance is known from the quadrature-axis identification. One possible way to determine the field winding resistance is by a stepwise excitation of $u_{f}$ and measuring $i_{f}$. Subsequently, dividing the steady-state value of $u_{f}$ by the the steady-state value of $i_{f}$ gives $R_{f}$.

Both the direct-axis winding flux $\psi_{d}$, and the field winding flux $\psi_{f}$, however, can not be measured in practice. Conversely, these variables can be generated by integration of Eq. (3) with the $u_{d}, u_{f}, i_{d}$, and $i_{f}$ acting as input. Analogous to the quadrature-axis identification, the latter variables can be deduced from the three measurable variables $u_{b}, u_{c}$, and $i_{c}$.

\section{Standstill test (excitation signal) selection}

It is straightforward to understand that the character of the input signal that is applied during the experiment highly determines the amount of relevant information that is present in the data. For example, applying a constant input signal $u(t)=c, t \in(-\infty, \cdots,+\infty)$ ( $c$ a constant) to the generator will not result in an output signal that contains any information on the dynamics of the system. Observe that in this case only static behaviour can be uniquely determined. Consequently, in order to extract sufficient information from measured data concerning the dynamics, conditions have to be imposed on the character of the input signal. 
In the derivation of Eqs. (1) and Eqs. (2) a linear magnetic circuit was assumed. Due to hysteresis and saturation, however, the relationship between the current $i$ and flux $\psi$ is nonlinear. As a result, the kind of excitation signal as well as the values of the currents during the measurements influence the identified slope of the hysteresis loop ${ }^{10}$. Fig. 3 shows two exaggerated hysteresis loops. The minor loop, which is symmetric with respect to the origin and has a smaller overall slope than the full loop, is traveled by small sinusoidal (as in the SSFR test) and random excitation signals. The only way to force the slope of the minor loop to approximate that of the full loop is to use large excitation signal amplitudes. Obviously, this requires special test equipment what is in conflict with our practical demands.

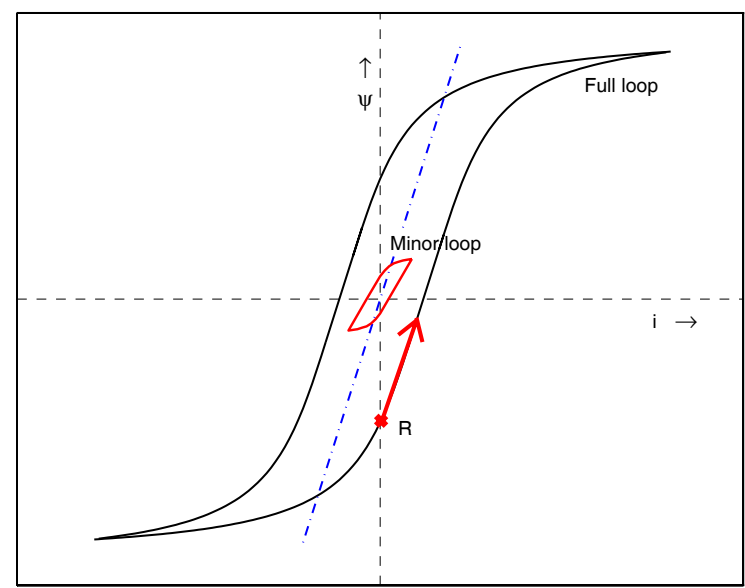

Figure 3: Full and minor hysteresis loop (width of loop is exaggerated) with approximate slope (dashed-dotted line) and R: initial magnetic state.

The slope of step or ramp excitation signals with the initial magnetic state fixed on the low boundary of the hysteresis loop (location R in Fig. 3) by preliminary magnetisation of the magnetic circuit, on the other hand, coincides with the full loop slope. In addition, while a complete SSFR test requires a long period of time, the step- or ramp-response test can be performed in a very short time period ${ }^{5}$. Consequently, step or ramp excitation signals are preferred over small sinusoidal and random excitation signals for our intended model use. The step excitation signal can be easily generated by switching on a low-power DC voltage source (e.g. an ordinary car battery). That is, it has the advantage of not requiring any special test equipment. Consequently step-response testing may be more practical for obtaining parameters for installed synchronous machines. Summarising, based on both the excitation requirements and the practical demands, a step-response test seems the most appropriate test among the standstill tests for the identification and parameter determination of the Lagerwey LW-50/750 generator.

Step-response tests are proposed by e.g. Boije et al. ${ }^{1}$, by Keyhani et al. ${ }^{5}$ and by Vleeshouwers ${ }^{17}$. The measurement set-up as well as the experimental procedures are sim- ilar in the aforementioned step-response tests. In all cases, a sudden DC voltage is applied across two of the stator terminals with the rotor positioned in the $d$ or $q$ axis. On the contrary, the way the parameters are identified is quite distinct. For example, Keyhani et al. obtain initial values for the maximum-likelihood estimation by first applying a curve-fitting procedure to measured data. Subsequently, the maximum-likelihood estimation algorithm is used to identify the $d$ and $q$ axis transfer function model parameters. Vleeshouwers, on the other hand, transforms the measured time-domain data first to the frequency domain and after that identifies the transfer function model parameters using a maximum-likelihood (ML) estimator. We have decided to follow the well-documented procedures of Vleeshouwers' "modified step-response test" (MSR) to generate the timedomain data. For identifying the transfer functions, however, we developed a new, straightforward procedure using the aforementioned variables.

\section{MSR TEST APPLIED TO LW-50/750 GENERATOR}

In the previous section the modified step-response (MSR) test turned out to be most appropriate for synchronous machine identification and parameter determination. In this section the MSR-test will be used to identify the transfer function $Y_{q}(s)$, the MIMO transfer function between the fluxes $\psi_{d}, \psi_{f}$ and the currents $i_{d}, i_{f}$ as well as the resistances $R_{s}$ and $R_{f}$ of the Lagerwey LW-50/750 generator. This generator is a $750 \mathrm{~kW}$, directly driven (or low-speed), salient-pole rotor synchronous generator. The power is generated in two star connected three-phase systems. A rectifier at the tower base converts the three-phase alternating currents to $1100 \mathrm{~V}$ DC of the DC-bus. Adjacent to the rectifier, is an inverter, which converts the DC to $690 \mathrm{~V}$ AC 50- $\mathrm{Hz}$ of the utility grid.

\section{A. Measurement set-up}

Fig. 4 shows a schematic of the measurement set-up. The whole set-up has been installed in the nacelle at about 50 meter above the ground level. The step-like excitation signal is generated by switching on a low-power DC voltage source (i.e. a $12 \mathrm{~V}$ battery). The battery is connected to the $b$ and $c$ stator terminals of the synchronous generator (SG). For the switching a thyristor is applied. A thyristor is preferred above a mechanical switch because it eliminates the problem of bouncing ${ }^{17}$. Furthermore, the machine parameters are identified at ambient temperature $\left(20^{\circ}\right)$.

Depending on the measurement type, a combination of the following signals is measured: $i_{c}$ (stator current), $u_{b c}$ (stator voltage), $u_{f}$ (field winding voltage), and $i_{f}$ (field winding current).

The data-acquisition system consists of three main parts, viz. an input-output (I/O) board, a digital signal processor board from dSPACE ${ }^{\circledR 2}$ with a TMS320C40 processor from Texas Instruments ${ }^{\circledR}$, and a personal computer (PC) connected to the processor board. The automated data-acquisition pro- 
cess is started by switching on the (mechanical) switch and subsequently triggering the thyristor.

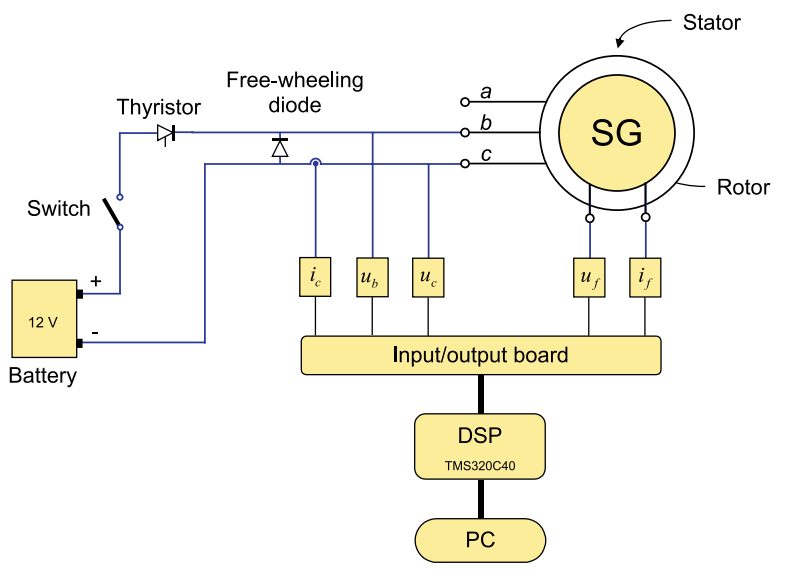

Figure 4: Scheme of the measurement set-up of the modified stepresponse test as used for the identification of the machine parameters of the synchronous generator $(S G)$ of the Lagerwey $L W-50 / 750$ wind turbine.

\section{B. Data-acquisition and identification procedure}

The modified step-response test consists of three successive measurements:

1. Q-measurement. Rotor positioned such that the quadrature axis is excited;

2. D-measurement. Rotor positioned such that the direct axis is excited, while the field winding is shortcircuited;

3. $R_{f}$-measurement. Stepwise excitation of $u_{f}$ and measuring $i_{f}$;

The "D" and "Q"- measurements have been preceded by remagnetising the system by either a large negative or positive current depending of the direction of the step in order to fix the initial magnetic state on the low boundary of the hysteresis loop (location R in Fig. 3).

It should be noted that none of the measurements incorporated anti-aliasing filters. The "Q"-measurement data were collected with a sample rate of $5 \mathrm{kHz}$ and have a 3.1 second measurement period. Both the " $\mathrm{D}$ " and " $R_{f}$ "-measurement data were collected with a sample rate of $1 \mathrm{kHz}$ and a 10.1 second measurement period. Each measurement is repeated at least three times. Example input output data of the "Q"measurement is shown in Fig. 5. Notice that the measured voltage appears to be a modified step instead of an exact step due to the battery's internal voltage drop.

\section{Parameter estimation procedure}

System identification or parameter estimation deals with constructing mathematical models of dynamical systems
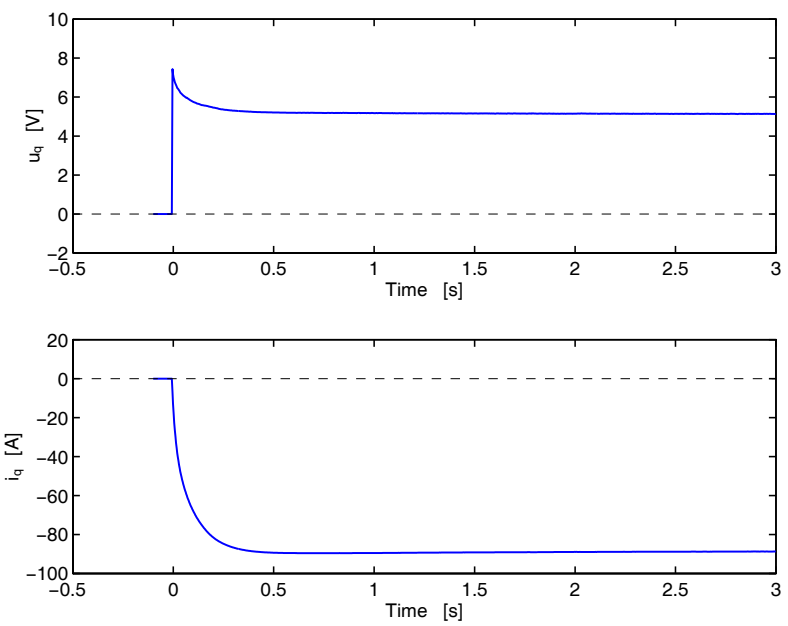

Figure 5: Time-domain MSR input-output signals for estimation of transfer function $Y_{q}(s)$ (excitation $u_{q}$ and response $i_{q}$ ).

from experimental data. The parameter estimation procedure picks out the "best" model within the chosen model structure according to the measured input and output sequences and some identification criterion. A common and general method of estimating the parameters in system identification is the prediction-error method ${ }^{8}$. In this method, the parameters of the model are chosen so that the difference between the model's (predicted) output and the measured output is minimized.

\section{C1. Black-box model structures}

In the system identification approach ${ }^{8}$, it is assumed that the "true" system description is given in the following form

$$
y(k)=G_{0}(q) u(k)+v(k)
$$

where $y(k)$ is the (measured) output signal, $G_{0}(q)$ is a proper, rational, stable transfer function, $q$ the shift operator, $u(k)$ the (measured) input signal, and $v(k)$ the disturbance signal. The disturbance $v(k)$ is modeled as a filtered sequence of zero-mean, identically distributed, independent random variables (i.e. white noise $e(k)$ )

$$
v(k)=H_{0}(q) e(k)
$$

The transfer function $H_{0}(q)$ is restricted to be monic $\left(H_{0}(0)=1\right)$ and minimum phase (i.e. $H_{0}^{-1}(q)$ has a stable inverse). The whole system specification is thus given by specifying the two transfer functions (or filters) $G_{0}(q)$ and $H_{0}(q)$.

Analogous to the true system description given by Eq. (9), the model is determined by the relation

$$
y(k, \theta)=G(q, \theta) u(k)+H(q, \theta) e(k)
$$

A particular model corresponds thus to the specification of $G(q, \theta)$ and $H(q, \theta)$. One way to parametrize the transfer functions $G(q, \theta)$ and $H(q, \theta)$ is to represent them as rational functions and let the parameters be the numerator and 
denominator coefficients. These coefficients are collected in the parameter vector $\theta$, which is to be estimated.

For single-input, single-output systems, the general linear, time invariant, black-box model structure is given by

$$
A(q) y(k)=q^{-n_{k}} \frac{B(q)}{F(q)} u(k)+\frac{C(q)}{D(q)} e(k)
$$

where $A, B, C, D$, and $F$ are polynomials in the delay operator $q^{-1}$

$$
\begin{aligned}
& A(q)=1+a_{1} q^{-1}+\cdots+a_{n_{a}} q^{-n_{a}} \\
& B(q)=b_{0}+b_{1} q^{-1}+\cdots+b_{n_{b}} q^{-n_{b}} \\
& C(q)=1+c_{1} q^{-1}+\cdots+c_{n_{c}} q^{-n_{c}} \\
& D(q)=1+d_{1} q^{-1}+\cdots+d_{n_{d}} q^{-n_{d}} \\
& F(q)=1+f_{1} q^{-1}+\cdots+f_{n_{f}} q^{-n_{f}}
\end{aligned}
$$

The numbers $n_{a}, n_{b}, n_{c}, n_{d}$, and $n_{f}$ are the orders of the respective polynomials. The number $n_{k}$ is the pure time delay (the dead-time) from input to output. Notice that for a sampled data system, $n_{k}$ is equal to 1 if there is no deadtime.

Within the structure of Eq. (12) all the usual linear black-box model structures are obtained as special cases. For example, the ARX (Autoregressive with external input) model structure is obtained for $n_{c}=n_{d}=n_{f}=0$.

\section{C2. One-step-ahead prediction error}

A model obtained by identification can be used in many ways, depending on the intended use of the model. For both simulation as well as control design purposes, it is valuable to know at time $(k-1)$ what the output of the system is likely to be at time $k$ in order to determine the input at time $(k-1)$. Therefore, the parameter estimate $\theta$ is usually determined so that the one-step-ahead prediction error

$$
\epsilon(k, \theta) \equiv y(k)-\hat{y}(k \mid k-1, \theta) \quad k=1, \cdots, N
$$

is small for every time instant. In Eq. (13) $\hat{y}(k \mid k-1, \theta)$ denotes the one-step-ahead prediction of $y(k)$ given the data up to and including time $(k-1)$ based on the parameter vector $\theta$. Observe that the prediction error can only be calculated a posteriori, when measurement $y(k)$ has become available. In Ljung ${ }^{8}$ it is shown that the one-step-ahead prediction of $y(k)$ is given by

$$
\begin{aligned}
\hat{y}(k \mid k-1, \theta) & =H^{-1}(q, \theta) G(q, \theta) u(k) \\
& +\left[1-H^{-1}(q, \theta)\right] y(k) \quad k=1, \cdots, N
\end{aligned}
$$

Recall that $H^{-1}(0, \theta)=1$, which means that the predictor depends only on previous output values. Substituting Eq. (14) in Eq. (13), the prediction error becomes

$$
\epsilon(k, \theta)=H^{-1}(q, \theta)[y(k)-G(q, \theta) u(k)]
$$

The prediction error is thus exactly that component of $y(k)$ that could not have been predicted at time instant $(k-1)$.
Obviously, in case of a consistent model estimate (i.e. if the estimated model $G(q, \theta), H(q, \theta)$ is equal to the true system $G_{0}(q), H_{0}(q)$ ), then the prediction error becomes a white noise signal $(\epsilon(k)=e(k))$.

\section{C3. Identification criterion}

The most simple and most frequently applied identification criterion is a quadratic function on $\epsilon(k, \theta)$, denoted as

$$
V_{N}\left(\theta, Z_{N}\right)=\frac{1}{N} \sum_{k=1}^{N} \epsilon^{2}(k, \theta)
$$

where $Z_{N}:=\{y(1), u(1), y(2), u(2), \cdots, y(N), z(N)\}$.

The estimated parameter vector $\hat{\theta}_{N}$ is now defined as the minimizing element of the criterion Eq. (16), i.e.

$$
\hat{\theta}_{N}=\arg \min _{\theta} V_{N}\left(\theta, Z_{N}\right)
$$

This criterion is known as the "least squares criterion".

For the FIR (finite impulse response; $n_{a}=n_{c}=n_{d}=$ $n_{f}=0$ ) and the ARX model structure the one-step-ahead prediction $\hat{y}(k \mid k-1)$ is a linear function of the polynomial coefficients that constitute the parameter vector $\theta$ (the socalled linear-in-the-parameters property). A consequence of this linearity is that a least squares identification criterion defined on the prediction errors $\epsilon(k)$ is a quadratic function in $\theta$. As a result, there will be an analytical expression for the optimal parameter $\hat{\theta}$ that minimizes the quadratic criterion. For all other model structures, on the other hand, the parameter estimation involves an iterative, numerical search for the best fit.

The developed identification procedure consists of three successive steps:

- Step 1: Pretreatment of data. In general, when the data have been collected from the identification experiment, they have to be pretreated to avoid problems during the parameter identification. The necessary pretreatment of the time-domain data, however, is limited since the MSR-test results in relatively clean signals. This is mainly because the machine has been taken out of operation. The only pretreatment of the data that is required is compensating for the (slight) static nonlinearity of the sensors, and removal of the offset;

- Step 2: Model structure and order selection. It is trivial that a bad model structure cannot offer a good, low order model, regardless the amount and quality of the available data. The measured input-output data is imported into SITB (graphical user interface to the System Identification Toolbox) ${ }^{14}$. First, an initial model order estimate is made by estimating 1100 ARX-models.

Recall that a linear regression estimate ARX is generally the most simple model to start with, particularly because of its computationally simplicity ${ }^{8}$. If the 
resulting model, however, produces an unsatisfactory simulation error and/or if the input is correlated with the residual, the model is rejected. Next, another model structure (or order) is selected until the model produces a satisfactory simulation error as well as results in zero cross-covariance between residual and past inputs. In that case it can be concluded that a consistent model estimate has been obtained;

- Step 3: Model validation. Model validation is highly important when applying system identification. The parameter estimation procedure picks out the "best" model within the chosen model structure. The crucial question is whether this "best" model is "good enough" for the intended application: time-domain simulation, analysis of dynamic loads, and control design purposes. To this end, the identified models should be confronted with as much information about the process that is practical. Here, the outputs of the identified model are compared to the measured ones on a data set that was not used for the fit (the so-called "validation data set").

\section{Model validation}

As mentioned above, the outputs of the identified model are compared to the measured ones from a validation data set to (in)validate the model. The percentage of the output variations that is reproduced by the model is chosen as measure of the "goodness" of fit. The precise definition is:

$$
M_{g \circ f}=\left[1-\frac{\sqrt{\sum_{t=-0.1}^{10}\left(y(t)-y_{s i m}(t)\right)^{2}}}{\sqrt{\sum_{t=-0.1}^{10}|y(t)-\bar{y}|^{2}}}\right] \cdot 100
$$

with $0 \leq M_{g o f} \leq 100 \%$ and $y$ is the measured output, $y_{\text {sim }}$ is the simulated model output, and $\bar{y}$ the average value.

\section{E. Results}

The $q$-axis transfer function $Y_{q}(s)$ of the electromagnetic part of the Lagerwey LW-50/750 generator has been identified using an ARX model structure. A third order model turned out to be sufficient. The $q$-axis parameters are derived from $Y_{q}(s)$ as outlined above.

The structure of the symmetric $d$-axis transfer function matrix (i.e. common denominator) and the high signal-to-noise ratio calls for a MIMO ARX model structure. For both inputs $i_{d}$ and $i_{f}$, the percentage of the variations in the fluxes $\psi_{d}, \psi_{f}$ that is reproduced by the (fourth order) model is larger than $99.5 \%$ (identification data set).

The aforementioned $q$-axis parameters and $d$-axis transfer function matrix as well as the field-winding resistance are implemented in the block diagram shown in Fig. 2. The resulting inputs and outputs of the model are shown in Fig. 6 for a validation data set. Obviously, the simulated data matches the measured data very well. Observe that $u_{f}$ is not equal to zero because the short-circuit is not perfect due to the slip-rings. Fig. 7 shows the outputs once again, but now on a reduced time scale. The percentage of the output variations that is reproduced by the model is in case of the identification data set $99.74 \%, 99.47 \%$, and $99.89 \%$ for $i_{d}$, $i_{f}$, and $i_{q}$ respectively. For the validation data set the percentages are $99.16 \%, 92.78 \%$, and $99.76 \%$ for $i_{d}, i_{f}$, and $i_{q}$ respectively.
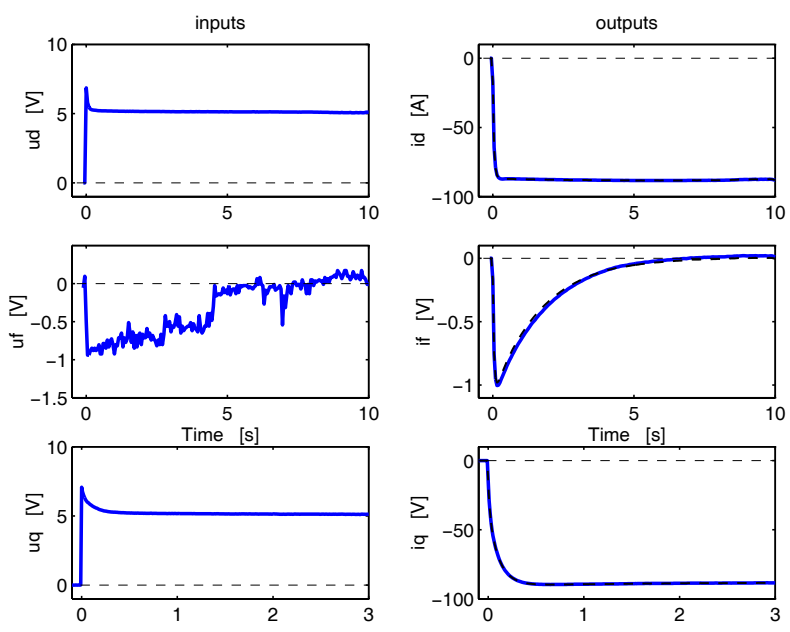

Figure 6: Left figures: measured inputs $u_{d}, u_{f}$ and $u_{q}$ as function of time. Right figures: outputs $i_{d}, i_{f}$ and $i_{q}$ as function of time. Solid lines: measured data (validation data set), and dashed-lines: simulated data.
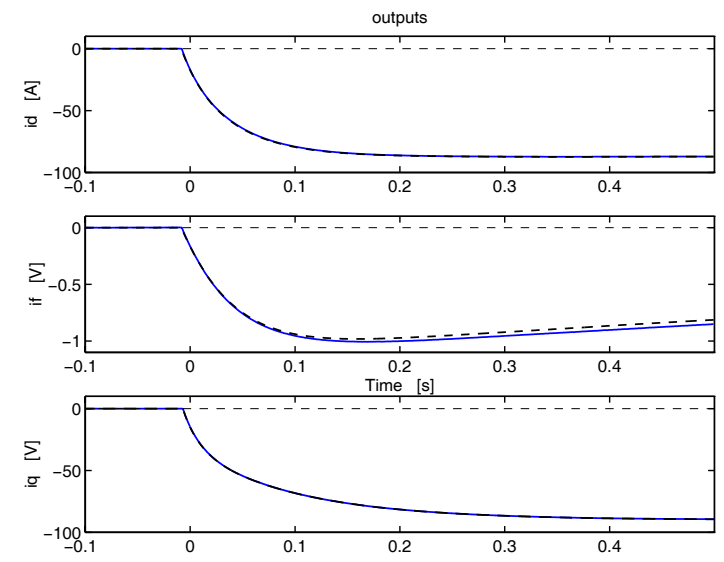

Figure 7: Outputs $i_{d}, i_{f}$ and $i_{q}$ as function of time. Solid lines: measured data (validation data set), and dashed-lines: simulated data.

In addition, the quality of the model is also checked by examining the cross correlation function between inputs and output residuals. In both cases an almost zero crosscovariance existed.

\section{CONCLUSIONS AND FUTURE WORK}

In this paper a new procedure for identifying the transfer functions of Park's $d q$-axis model of a synchronous generator has been developed. The following conclusions can be drawn. 


\section{Conclusions}

A theoretical model of the electromagnetic part of a synchronous generator has been proposed. It has been shown that the parameters of this model can be easily identified following the developed procedure. The required input-output data is obtained from the modified step-response test. This test is the most favourable standstill test considering equipment costs and weight, measurement time and complexity.

The validity of the theoretical model has been verified by comparing time-domain simulations with measurements taken from the Lagerwey LW-50/750 generator.

Combination of the aforementioned results leads to the conclusion that it is possible to identify an accurate model of the electromagnetic part of the Lagerwey LW-50/750 generator on the basis of modified step-response data. Ultimate validation, however, will follow after the implementation of the designed frequency converter controller in the Lagerwey LW-50/750 wind turbine.

\section{Future work}

The validated model will be used to develop a new, robust frequency converter controller for high dynamic performance of the Lagerwey LW-50/750 wind turbine directdrive synchronous generator.

\section{ACKNOWLEDGEMENTS}

The authors would like to thank André Pubanz of EMechForce and Peter Valk of the Mechanical Engineering Systems and Control group of Delft University of Technology for their support during the performed standstill generator measurements.

\section{REFERENCES}

[1] E.S. Boije, J.C. Balda, R.G. Harley, and R.C. Beck, "Time-domain identification of synchronous machine parameters from standstill tests.", In IEEE Trans. on Energy Conversion, Vol. 5, No. 1, pp. 164-175, 1990.

[2] dSPACE ${ }^{\circledR}:$ http://www.dspace.de/

[3] S. Henschel, and H.W. Dommel, "Noniterative Synchronous Machine Parameter Identification from Frequency Response Tests.”, In IEEE Trans. on Power Systems, Vol. 14, No. 2, pp. 553-560, 1999.

[4] IEEE Standard 115-1995: IEEE Guide: Test Procedures for Synchronous Machines Part I - Acceptance and Performance Testing and Part II - Test Procedures and Parameter Determination for Dynamic Analysis, Institute of Electrical and Electronics Engineers, Inc. New York, NY, 216p., 1996.

[5] A. Keyhani, H. Tsai, and T. Leksan, "Maximum likelihood estimation of synchronous machine parameters from standstill time response data.", In IEEE Trans. on Energy Conversion, Vol. 9, No. 1, pp. 98-114, 1994.
[6] P. Kundur, Power System Stability and Control, McGraw-Hill, Inc., 1176p., 1994.

[7] L.X. Le, and W.J. Wilson, "Synchronous machine parameter identification: a time-domain approach.", In IEEE Trans. on Energy Conversion, Vol. 3, No. 2, pp. 241-248, 1988.

[8] L. Ljung, System Identification - Theory for the User. Prentice-Hall, Englewood Cliffs, New Jersey, 519p., 1987.

[9] D-P. Molenaar and Sj. Dijkstra, Modeling the structural dynamics of the Lagerwey $L W-50 / 750$ wind turbine. In Wind Engineering, Vol. 22, No. 6, pp. 253-264, 1998.

[10] S.H. Minnich, "Small signals, large signals, and saturation in generator models." In IEEE Transactions on Energy Conversion, Volume EC-1, No. 1, pp. 95-103, March 1986.

[11] R.H. Park, "Two-Reaction Theory of Synchronous Machines, Generalized Method of Analysis." Part I: In AIEE Transactions, Vol. 48, pp. 716-730, July 1929.

[12] R.H. Park, "Two-Reaction Theory of Synchronous Machines, Generalized Method of Analysis.” Part II: In AIEE Transactions, Vol. 52, pp. 352-355, June 1933.

[13] R.M. Saunders, "Synchronous-machine standstill frequency-response test data analysis", In IEEE Trans. on Energy Conversion, Vol. 6, No. 3, pp. 564-571, 1991.

[14] System Identification Toolbox User's Guide. The MathWorks, Inc. August 1995.

[15] O. Touhami, H. Guesbaoui, and C. Iung:, "Synchronous Machine Parameter Identification by a Multitime Scale Technique." In IEEE Trans. on Industrial Applications, Vol. 30, No. 6, pp. 1600-1608, 1994.

[16] A. Tumageanian, and A. Keyhani, "Identification of synchronous machine linear parameters from standstill step voltage input data.", In IEEE Trans. on Energy Conversion, Vol. 10, No. 2, pp. 232-240, June 1995.

[17] J.M. Vleeshouwers, Synchronous machine identification by a simple step-response test. $\mathrm{PhD}$. Thesis, Eindhoven University of Technology, April 1998. 